\title{
Cut-Down Method in the Inductive Limit Decomposition of Non-Commutative Tori, III: A Complete Answer in 3-Dimension
}

\author{
Qing Lin ${ }^{1,2}$ \\ 1 Institute of Mathematics, Chinese Academy of Sciences, People's Republic of China \\ 2 Department of Mathematics and Statistics, University of Victoria, Victoria, British Columbia, \\ Canada V8W 3P4
}

Received: 26 May 1995 / Accepted: 13 November 1995

\begin{abstract}
We use the cut-down method and a multi-dimensional continued fraction approximation to prove that any simple 3-torus is an inductive limit of direct sums of four circle algebras. Consequently, simple 3-tori are classified by the ordered $K_{0}$-group with distinguished order unit.
\end{abstract}

\section{Introduction}

A non-commutative $n$-torus is the universal $\mathrm{C}^{*}$-algebra generated by $n$ unitaries, $U_{1}, U_{2}, \ldots, U_{n}$, with non-trivial linear commutation relations

$$
U_{j} U_{i}=\left(\exp \left(2 \pi i \theta_{i j}\right)\right) U_{i} U_{j}, \quad 1 \leqq i<j \leqq n
$$

where $\theta_{i j}$ is a real number. We shall call such unitary generators canonical and a simple non-commutative $n$-torus a simple $n$-torus.

Such a $C^{*}$-algebra is often represented as a twisted group $C^{*}$-algebra of $\mathbf{Z}^{n}$, with respect to a bicharacter $\beta$, and written as $\mathrm{C}^{*}\left(\mathbf{Z}^{n}, \beta\right)$. The two definitions may be identified as follows:

Let $e_{1}, \ldots, e_{n}$ be a basis of $\mathbf{Z}^{n}$, and denote by $\chi_{e_{j}}$ the characteristic function on $\mathbf{Z}^{n}$ supported at $e_{j}$; if we write $\beta\left(e_{i}, e_{j}\right) \overline{\beta\left(e_{j}, e_{i}\right)}$ in the form $\exp \left(-2 \pi i \theta_{i j}\right)$, we can identify $\chi_{e_{j}}$ with $U_{j}$ as in $(* 1)$, for $1 \leqq j \leqq n$.

We shall feel free to use both definitions and this identification.

For the importance of this class of $\mathrm{C}^{*}$-algebras, we refer to [R4, $\mathrm{P}$ and Po].

Built on [R3, EE, EL1 and EL2], we are now able to show that every simple 3 -torus is an inductive limit of direct sums of four circle algebras. Consequently (applying Corollary 1 of the main theorem in [Po], also see [Pa] and the appendix of our paper for a direct proof), any non-commutative 3-torus is an inductive limit of type I $\mathrm{C}^{*}$-algebras.

Combining the classification theorem of Elliott (Theorem 7.1 of [E]), we see that the ordered $K_{0}$-group with a distinguished order unit is a complete isomorphic 
invariant for a simple 3-torus. Consequently, a complete stably isomorphic invariant for an arbitrary 3-torus is the ordered $K_{0}$-group, or equivalently, the range of the canonical trace on its $K_{0}$-group, up to a scalar multiple. Notice that such a $\mathrm{C}^{*}$-algebra may neither be simple nor real rank zero.

This answers an open question asked ten years ago (see [BCEK]).

Part of the results here was reported in December 1994, at the Fields Institute Workshop "Classification of Amenable C*-Algebras."

We shall outline the idea of the proof.

Let $A$ be a non-commutative 3-torus with canonical generators $U_{1}, U_{2}, U_{3}$ and associated commutation parameters $\theta_{i j}$ 's. In the paper [EL2], we prove that if $A$ is simple and $\left(1, \theta_{12}, \theta_{13}, \theta_{23}\right)$ are rationally dependent, then $A$ is an inductive limit of direct sums of four circle algebras. Therefore in this paper, we will only consider the case where $\left(1, \theta_{12}, \theta_{13}, \theta_{23}\right)$ are rationally independent. Note that this condition itself implies the simplicity of $A$.

Our solution to this problem consists of two steps.

In general, let $S$ be a finite subset of a unital $C^{*}$-algebra $B$. To approximate $S$ by a unital $C^{*}$-subalgebra of $B$ of a special type (e.g. direct sums of circle algebras) up to $\delta$, we might try the following naive idea. If a projection $e$ in $B$ satisfies the following condition:

then

$$
\left\|\left[e, x_{j}\right]\right\|<\frac{\delta}{4}, \quad \text { for all } x_{j} \text { in } S
$$

$$
\left\|x_{j}-\left(e x_{j} e \oplus(1-e) x_{j}(1-e)\right)\right\|<\frac{\delta}{2}, \quad \text { for all } x_{j} \text { in } S
$$

Therefore the above approximation problem reduces to two similar problems for $e S e$ in $e B e$ and for $(1-e) S(1-e)$ in $(1-e) B(1-e)$. If, in addition, $e S e$ and $(1-e) S(1-e)$ are both nearly "half" of $S$, we have a better chance to solve the two reduced problems. The difficulty lies in understanding the word "half" in a correct way and formulating a nice sufficient condition on $e$ which leads to such a situation.

Let $A$ be a simple 3 -torus as above such that $\left(1, \theta_{12}, \theta_{13}, \theta_{23}\right)$ are rationally independent, and $S=\left\{U_{1}, U_{2}, U_{3}\right\}$. In this case, we may choose the rank of the corresponding $K_{1}$-classes to measure the size "half." Thus our first step is, for a sufficiently large class of approximately central projections, formulating a nice sufficient condition which leads to the desired situation. We do it in the following way. At first, for a projection $e$ approximately commuting with all $U_{j}$ 's up to $\varepsilon$ and in the class which we shall specify in the next section, we study the approximate position of $e S e$ in $e A e$ and that of $(1-e) S(1-e)$ in $(1-e) A(1-e)$, up to a universal multiple of $\sqrt{\varepsilon}$. We achieve this in a local reduction theorem by a suitable strong Morita equivalence construction (cf. [R3 and EL1]). Then by considering $\left\{\left[e U_{j} e\right]_{1}: j=1,2,3\right\}$ in $K_{1}(e A e)$ and $\left\{\left[(1-e) U_{j}(1-e)\right]_{1}: j=\right.$ $1,2,3\}$ in $K_{1}((1-e) A(1-e))$, we are able to formulate a nice sufficient condition which leads to an approximation of $S$ by direct sums of four circle algebras in $A$. We formulate this condition as a non-standard Diophantine approximation to $\left(1, \theta_{12}, \theta_{13}, \theta_{23}\right)$.

The second step is to solve this non-standard Diophantine approximation problem. We achieve this by a natural modification of the ordinary strongly convergent multi-dimensional continued fraction approximation. 


\section{The Local Reduction Theorem}

In this section, we will assume that $A$ is a simple non-commutative 3-torus with canonical generators $U_{1}, U_{2}$ and $U_{3}$. Also we assume the rational independence of $\left(1, \theta_{12}, \theta_{13}, \theta_{23}\right)$.

We will first specify a class of projections that we are going to study.

Definition 1. Let $W_{1}$ and $W_{2}$ be two unitaries in a $\mathrm{C}^{*}$-algebra with the nontrivial linear commutation relation:

$$
W_{1} W_{2}=(\exp 2 \pi i \theta) W_{2} W_{1},
$$

where $0<\theta<1 / 2$. We call the following projection the normalized Rieffel projection generated by the ordered pair $\left(W_{1}, W_{2}\right)$ :

$$
e\left(W_{1}, W_{2}\right)=\check{F}_{0}\left(W_{1}\right)+\check{G}_{0}\left(W_{1}\right) \cdot W_{2}+\left(\check{G}_{0}\left(W_{1}\right) \cdot W_{2}\right)^{*},
$$

where $\check{F}_{0}$ and $\check{G}_{0}$ are two continuous functions on $\mathbf{R} / \mathbf{Z}$ defined as follows. In the interval $[0,1]$, the support of $\breve{F}_{0}$ is $[1 / 2-\theta, 1 / 2+\theta]$, the function $\breve{F}_{0}$ takes 1 at $1 / 2$ and linear in the remaining subintervals; the function $\check{G}_{0}$ equals $\sqrt{\check{F}_{0}\left(1-\check{F}_{0}\right)}$ on $[1 / 2,1 / 2+\theta]$ and zero otherwise.

Notice that, if $\theta$ is irrational and $\tau$ is the canonical trace on $\mathrm{C}^{*}\left(W_{1}, W_{2}\right)$ which annihilates the set $\left\{W_{1}^{m} W_{2}^{n}:(m, n) \neq(0,0)\right\}$, then $\tau\left(e\left(W_{1}, W_{2}\right)\right)=\theta$.

Let $\vec{\mu}=\left(\mu_{1}, \mu_{2}, \mu_{3}\right)$ be an integral vector. We denote by $U^{\vec{\mu}}$ the unitary $U_{1}^{\mu_{1}} U_{2}^{\mu_{2}}$ $U_{3}^{\mu_{3}}$ in $A$. Let $\vec{v}$ be another integral vector, then

$$
U^{\vec{v}} U^{\vec{\mu}}=(\exp 2 \pi i\langle\vec{\mu} \times \vec{v}, \vec{\Theta}\rangle) U^{\vec{\mu}} U^{\vec{v}},
$$

where $\vec{\Theta}=\left(\theta_{23},-\theta_{13}, \theta_{12}\right)$ and $\vec{\mu} \times \vec{v}$ is the classical vector cross product in $\mathbf{R}^{3}$.

For the vector $\vec{v}$ which is 1 at the $j$-th component and zero otherwise, we have that $\langle\vec{\mu} \times \vec{v}, \vec{\Theta}\rangle=-\langle\vec{\mu} \times \vec{\Theta}, \vec{v}\rangle=-(\vec{\mu} \times \vec{\Theta})_{j}$. This is exactly the commutation parameter of $U_{j}$ with $U^{\vec{\mu}}$. We have the following easy estimate.

Lemma 1. Let $(\vec{\mu}, \vec{v})$ be a pair of rationally independent integral vectors and $\varepsilon$ be a small positive number. If for the Euclidean distance,

$$
\operatorname{dist}\left(\vec{\mu} \times \vec{\Theta}, \mathbf{Z}^{3}\right)<\varepsilon \text { and } \operatorname{dist}\left(\vec{v} \times \vec{\Theta}, \mathbf{Z}^{3}\right)<\varepsilon,
$$

and if the fractional part $\theta$ of $\langle\vec{\mu} \times \vec{v}, \vec{\Theta}\rangle$ is less than $1 / 2$, then

$$
\left\|\left[e\left(U^{\vec{v}}, U^{\vec{\mu}}\right), U_{j}\right]\right\|<2 \pi \varepsilon+\frac{\varepsilon}{\theta}+4 \sqrt{\frac{\varepsilon}{\theta}}, \quad \text { for } j=1,2,3 .
$$

A weaker converse is true, but we will not need it.

Our special class of projections consists of all projections of the form $e\left(U^{\vec{v}}, U^{\vec{\mu}}\right)$, where $(\vec{\mu}, \vec{v})$ is a pair of integral vectors which are rationally independent and satisfy the following technical condition $(* 2)$.

If we write $\vec{\mu}=p \vec{\mu}_{1}$ and $\vec{v}=k \vec{v}_{1}$ such that $p, k$ are positive integers and $\vec{\mu}_{1}, \vec{v}_{1}$ are primitive integral vectors, then

(1) $\vec{\mu}_{1} \times \vec{v}_{1}$ is primitive; 
(2) $1 /(15 p)<\alpha:=a+k\left\langle\vec{\mu}_{1} \times \vec{v}_{1}, \vec{\Theta}\right\rangle<1 / 2 p$, where $-a$ is the integral part of $k\left\langle\vec{\mu}_{1} \times \vec{v}_{1}, \vec{\Theta}\right\rangle$

(3) $(1-p a) / k$ is an integer.

In the next section, we shall produce many integral and rationally independent vector pairs satisfying the above technical condition.

From now on, we will always assume that the pair $(\vec{\mu}, \vec{v})$ satisfies the above condition and use the notations $p, k, a, \alpha, \vec{\mu}_{1}, \vec{v}_{1}$ as defined in $(* 2)$. We will also denote by $e$ the projection $e\left(U^{\vec{v}}, U^{\vec{\mu}}\right)$ and use the symbol " " to mean "near."

Next, we study the approximate position of $\left\{e U_{j} e: j=1,2,3\right\}$ in $e A e$ and $\left\{(1-e) U_{j}(1-e): j=1,2,3\right\}$ in $(1-e) A(1-e)$, up to small distances.

Since the way we study $e A e$ is through a Morita equivalence $e A e-A$ bimodule of M.A. Rieffel (cf. [R3]), we need to smooth $e$ first.

Denote $\check{F}_{0} \cdot \chi_{[0,1]}$ by $\breve{f}_{0}$, where $\chi_{[0,1]}$ is the characteristic function on $[0,1]$ and $\check{F_{0}}$ is the continuous function in the definition of $e\left(U^{\vec{v}}, U^{\vec{\mu}}\right)$.

For each large number $N$, we define a non-negative Schwartz function $f_{0}$ with the same support as that of $\breve{f}_{0}$ and satisfying:

(1) $f_{0}(x+p \alpha)=1-f_{0}(x)$ and $f_{0}(1-x)=f_{0}(x), 1 / 2-p \alpha \leqq x \leqq 1 / 2-p \alpha / 2$;

(2) $\left\|\sqrt{f_{0}}-\sqrt{\check{f}_{0}}\right\|_{\infty}<1 / N$.

Then we define a smooth projection $e_{0}$ by the same formula as that of $e$, with $\check{F}_{0}, \check{G}_{0}$ replaced by

$$
F_{0}(x):=\sum_{n \in \mathbf{Z}} f_{0}(x+n) \quad \text { and } \quad G_{0}(x):=\sum_{n \in \mathbf{Z}} \sqrt{f_{0}(x+n) f_{0}(x+n-p \alpha)} .
$$

Clearly, when $N \rightarrow \infty$, we have $f_{0} \rightarrow \check{f_{0}}$ and $e_{0} \rightarrow e\left(U^{\vec{v}}, U^{\vec{\mu}}\right)$.

Also it is easy to check the following inequality

$$
\left|\sqrt{f_{0}(x)}-\sqrt{f_{0}(y)}\right| \leqq \frac{2}{N}+\sqrt{\frac{|x-y|}{p \alpha}}, \quad x, y \in \mathbf{R} .
$$

Definition 2. We say that a unitary generator $\left\{V_{0}, V_{1}, V_{2}, V_{3}\right\}$ of eAe is standard, if

(1) their commutation relations are all linear (as in $(* 1))$,

(2) the unitary $V_{0}$ has finite order.

Note that, if $A$ is simple, then $\mathrm{C}^{*}\left(V_{1}, V_{2}, V_{3}\right)$ must be a non-commutative 3-torus.

For a pair of rationally independent integral vectors $(\vec{\mu}, \vec{v})$ satisfying the above condition $(* 2)$, we prove the following crucial theorem.

The Local Reduction Theorem. With the notations above (including those introduced in $(* 2)$ ) and $0<\varepsilon<1 / 15$, suppose

and

$$
\operatorname{dist}\left(\vec{\mu} \times \vec{\Theta}, \mathbf{Z}^{3}\right)=\left\|\vec{\mu} \times \vec{\Theta}-\vec{Q}_{1}\right\|<\varepsilon
$$

$$
\operatorname{dist}\left(\vec{v} \times \vec{\Theta}, \mathbf{Z}^{3}\right)=\left\|\vec{v} \times \vec{\Theta}-\vec{Q}_{2}\right\|<\varepsilon,
$$

where $\vec{Q}_{1}$ and $\vec{Q}_{2}$ are two integral vectors. Then there is a standard unitary generator $\left\{V_{0}, V_{1}, V_{2}, V_{3}\right\}$ of eAe (where $\left.e=e\left(U^{\vec{v}}, U^{\vec{\mu}}\right)\right)$ and there is another one 
$\left\{\tilde{V}_{0}, \tilde{V}_{1}, \tilde{V}_{2}, \tilde{V}_{3}\right\}$ of $(1-e) A(1-e)$ such that, for $j=1,2,3$,

$e U_{j} e \sim a$ Laurent monomial in $V_{0}, V_{1}, V_{2}, V_{3}$, (up to a scalar multiple) multiple)

$(1-e) U_{j}(1-e) \sim$ a Laurent monomial in $\tilde{V}_{0}, \tilde{V}_{1}, \tilde{V}_{2}, \tilde{V}_{3}$, (up to a scalar all to within $16 \sqrt{\varepsilon}+17 \pi \varepsilon$.

Proof. At first, for a large number $N$, we smooth $e$ to a projection $e_{0}$ as above. One can check directly that

$$
\left\|\left[e_{0}, U_{j}\right]\right\| \leqq 2 \pi \varepsilon+\frac{\varepsilon}{\theta}+4 \sqrt{\frac{\varepsilon}{\theta}}+\frac{12}{N},
$$

for $j=1,2,3$. We will work with $e_{0}$ instead of $e$. At the end, the result will be passed from $e_{0}$ to $e$ by taking the limit as $N \rightarrow \infty$.

To study $e_{0} A e_{0}$ and $\left(1-e_{0}\right) A\left(1-e_{0}\right)$, we extend $\vec{\mu}_{1}, \vec{v}_{1}$ to an integral basis $\left\{\vec{\mu}_{1}, \vec{v}_{1}, \vec{\omega}_{1}\right\}$ of $\mathbf{Z}^{3}$ with

$$
\operatorname{det}\left(\vec{\mu}_{1}, \vec{v}_{1}, \vec{\omega}_{1}\right)=1 .
$$

Denote by $(\vec{m}, \vec{l}, \vec{n})$ the inverse matrix of $\left(\vec{\mu}_{1}, \vec{v}_{1}, \vec{\omega}_{1}\right)^{\top}$. Then it is clear that

$$
\vec{n}=\vec{\mu}_{1} \times \vec{v}_{1}, \quad \vec{n} \times \vec{m}=\vec{v}_{1} \quad \text { and } \quad \vec{l} \times \vec{n}=\vec{\mu}_{1} .
$$

After the papers [EL1 and EL2], the format of the following construction becomes standard. Therefore, we will sometimes sketch a routine calculation and refer to the above two sources for the detail.

Part One. The $e_{0} \mathrm{Ae}_{0}$ part.

Let us first take an Abelian group

$$
G=\left(\mathbf{R} \times \mathbf{Z} \times \mathbf{Z}_{p} \times \mathbf{Z}_{k}\right) \times\left(\mathbf{R} \times \mathbf{T} \times \mathbf{Z}_{p} \times \mathbf{Z}_{k}\right)
$$

twisted by the Heisenberg cocycle $\beta$ (cf. [R3]), and then a lattice $D=\operatorname{span}\left(\varepsilon_{1}, \varepsilon_{2}, \varepsilon_{3}\right)$ $\subseteq G$ defined by

$$
\left(\begin{array}{l}
\varepsilon_{1} \\
\varepsilon_{2} \\
\varepsilon_{3}
\end{array}\right)=\left(\begin{array}{llllllll}
x_{1} & n_{1} & m_{1} & l_{1} & y_{1} & z_{1} & 0 & l_{1}^{\prime} \\
x_{2} & n_{2} & m_{2} & l_{2} & y_{2} & z_{2} & 0 & l_{2}^{\prime} \\
x_{3} & n_{3} & m_{3} & l_{3} & y_{3} & z_{3} & 0 & l_{3}^{\prime}
\end{array}\right)=\left(\alpha \vec{m}, \vec{n}, \vec{m}, \vec{l}, \frac{1}{k} \vec{l}, \vec{z}, 0, a \vec{m}\right),
$$

where $\vec{z}=-\langle\vec{m}, \vec{\Theta}\rangle \vec{l}+\langle\vec{l}, \vec{\Theta}\rangle \vec{m}$.

From the identity

$$
\frac{\alpha}{k} \vec{m} \times \vec{l}+\vec{n} \times \vec{z}=\vec{\Theta}+\frac{a}{k} \vec{m} \times \vec{l},
$$

we have $\mathrm{C}^{*}(D, \bar{\beta})=A$, where $U_{j}$ is identified with $\chi_{\varepsilon_{j}} / p \alpha$.

M.A. Rieffel ([R3]) has shown that a suitable completion of the space of Schwartz functions on $\mathbf{R} \times \mathbf{Z} \times \mathbf{Z}_{p} \times \mathbf{Z}_{k}$ forms a strong Morita equivalence $\mathrm{C}^{*}\left(D^{\perp}, \beta\right)-\mathrm{C}^{*}(D, \bar{\beta})$ bimodule, where $D^{\perp}$ is the identified annihilator of $D$ through the anti-symmetrization of $\beta$, that is

$$
D^{\perp}=\{x \in G: \beta(x, y) \overline{\beta(y, x)}=1 \text {, for all } y \in D\} .
$$

We will show that $\mathrm{C}^{*}\left(D^{\perp}, \beta\right) \cong e_{0} A e_{0}$ in a nice way. 
Denote $(1-p a) / k$ by $s_{0}$. We claim that $D^{\perp}=\operatorname{span}\left(\delta_{0}, \delta_{1}, \delta_{2}, \delta_{3}\right)$, where

$$
\left(\begin{array}{c}
\delta_{0} \\
\delta_{1} \\
\delta_{2} \\
\delta_{3}
\end{array}\right)=\left(\begin{array}{cccccccc}
0 & 0 & {[1]_{p}} & 0 & 0 & 0 & 0 & 0 \\
-k\langle\vec{m}, \vec{\Theta}\rangle & -1 & 0 & 0 & \frac{-1}{\alpha}\langle\vec{l}, \vec{\Theta}\rangle & 0 & 0 & 0 \\
1 & 0 & 0 & 0 & 0 & 0 & 0 & {[1]_{k}} \\
0 & 0 & 0 & {[-1]_{k}} & \frac{-1}{p k \alpha} & 0 & {\left[s_{0}\right]_{p}} & 0
\end{array}\right) .
$$

It is easy to check that $\delta_{j}$ 's are all in $D^{\perp}$.

To see the converse, let $\varpi=\left(x, n,[m]_{p},[l]_{k}, y,[z]_{\mathbf{Z}},\left[m^{\prime}\right]_{p},\left[l^{\prime}\right]_{k}\right)$ be in $D^{\perp} \Longleftrightarrow m$ is arbitrary and

where $\xi_{j}$ 's are integers.

$$
\frac{1}{k} x \vec{l}-\alpha y \vec{m}-z \vec{n}+n \vec{z}+\frac{a l}{k} \vec{m}-\frac{m^{\prime}}{p} \vec{m}-\frac{l^{\prime}}{k} \vec{l}=(\vec{m}, \vec{l}, \vec{n})\left(\begin{array}{c}
\xi_{1} \\
\xi_{2} \\
\xi_{3}
\end{array}\right),
$$

$\Longleftrightarrow m$ is arbitrary and

$$
\left(\frac{1}{k} \vec{l},-\alpha \vec{m},-\vec{n}\right)\left(\begin{array}{c}
x \\
y \\
z+\xi_{3}
\end{array}\right)=\left(\vec{z}, \frac{1}{k} \vec{l}, \frac{1}{p k} \vec{m}\right)\left(\begin{array}{c}
-n \\
l^{\prime}+k \xi_{2} \\
k m^{\prime}-p a l+k p \xi_{1}
\end{array}\right) .
$$

Since

$$
\left(\frac{1}{k} \vec{l},-\alpha \vec{m},-\vec{n}\right)^{-1}=\left(k \vec{v}_{1},-\frac{1}{\alpha} \vec{\mu}_{1},-\vec{\omega}_{1}\right)^{\top}
$$

we deduce that

$$
\varpi=m \delta_{0}-n \delta_{1}+\left(l^{\prime}+k \xi_{2}\right) \delta_{2}+\left(-p a l+k m^{\prime}+k p \xi_{1}\right) \delta_{3} .
$$

We shall denote $\chi_{\delta_{j}}$ by $V_{j}$.

Define a Schwartz function $f$ on $\mathbf{R} \times \mathbf{Z} \times \mathbf{Z}_{p} \times \mathbf{Z}_{k}$ simply by setting $f(x, 0,0,0)=\sqrt{f_{0}(x)}(x \in \mathbf{R})$ and zero otherwise. Then a direct calculation as in [EL1] shows that

$$
\frac{1}{p \alpha}\langle f, f\rangle_{D^{\perp}}={ }^{C^{*}}{ }_{\left(D^{\perp}, \beta\right)} \text { and } \quad \frac{1}{p \alpha}\langle f, f\rangle_{D}=e_{0} .
$$

For the formula concerning the two $\mathrm{C}^{*}$-algebraic valued inner products $\langle,\rangle_{D^{\perp}}$ and $\langle,\rangle_{D}$, we refer to [EL1 or R3].

Now, we have a natural isomorphism $\Phi_{1}$ of $e_{0} A e_{0}$ onto $\mathrm{C}^{*}\left(D^{\perp}, \beta\right)$ defined by

$$
e_{0} c e_{0} \mapsto \frac{1}{p \alpha}\langle f * c, f\rangle_{D^{\perp}}
$$

We will identify the two $\mathrm{C}^{*}$-algebras via this map and then $\left\{V_{0}, V_{1}, V_{2}, V_{3}\right\}$ becomes a standard generator of $e_{0} A e_{0}$.

In a way similar to the computation in [EL1 and EL2], for each $j=1,2,3$ and $\varpi \in D^{\perp}$, we have

$$
\begin{gathered}
\left\langle f * U_{j}, f\right\rangle_{D^{\perp}}(\varpi)=0, \quad \text { unless } \\
\varpi=-m_{j} \delta_{0}+n_{j} \delta_{1}+t_{2 j} \delta_{2}+\left(l_{j}+t_{3 j} k\right) \delta_{3}=\left(x_{0 j}, \ldots, y_{0 j}, \ldots\right),
\end{gathered}
$$

where $t_{2 j}$ and $t_{3 j}$ are integers. 
Moreover, for such a $\varpi$,

$$
\begin{aligned}
\frac{1}{p \alpha}\left\langle f * U_{j}, f\right\rangle_{D^{\perp}}(\varpi)= & \frac{1}{p \alpha} \int_{\mathbf{R}} \sqrt{f_{0}(x) f_{0}\left(x+x_{0 j}+x_{j}\right)} \exp \left(-2 \pi i x\left(y_{j}+y_{0 j}\right)\right) d x \\
& \cdot \exp \left(-2 \pi i\left(x_{0 j} y_{j}-n_{j} z_{j}-\frac{l_{j} a m_{j}}{k}\right)\right),
\end{aligned}
$$

where

and

$$
\vec{x}_{0}=\left(\begin{array}{c}
x_{10} \\
x_{20} \\
x_{30}
\end{array}\right)=-k\langle\vec{m}, \vec{\Theta}\rangle \vec{n}+\vec{t}_{2},
$$

$$
\vec{y}_{0}=\left(\begin{array}{l}
y_{10} \\
y_{20} \\
y_{30}
\end{array}\right)=\frac{-\langle\vec{l}, \vec{\Theta}\rangle}{\alpha} \vec{n}-\frac{1}{p k \alpha}\left(\vec{l}+k \vec{t}_{3}\right) .
$$

Consequently, we get

$$
\begin{gathered}
\vec{x}_{0}+\vec{x}=\left(\vec{v} \times \vec{\Theta}-\vec{Q}_{2}\right)+\left(\vec{Q}_{2}+a \vec{m}+\vec{t}_{2}\right), \\
\vec{y}_{0}+\vec{y}=\frac{1}{p \alpha}\left(\left(\vec{Q}_{1}-\vec{\mu} \times \vec{\Theta}\right)+\frac{p a-1}{k} \vec{l}-\vec{Q}_{1}-\vec{t}_{3}\right) .
\end{gathered}
$$

Considering the support of $f_{0}$, it is clear that

$$
\left\langle f * U_{j}, f\right\rangle_{D^{\perp}}(\varpi)=0,
$$

unless $\vec{t}_{2}=-\left(\vec{Q}_{2}+a \vec{m}\right)+\vec{\Delta}$, where the components of $\vec{\Delta}$ are taken from $\{0, \pm 1\}$.

Let us denote by $Q_{j 1}$ and $Q_{j 2}$ the $j$-th component of $\vec{Q}_{1}$ and $\overrightarrow{Q_{2}}$ respectively. We can rewrite the above calculation in the following form:

$$
\begin{aligned}
\frac{1}{p \alpha}\left\langle f * U_{j}, f\right\rangle_{D^{\perp}}= & \xi_{j 0} H_{j 0}\left(V_{3}^{k}\right) V_{0}^{-m_{J}} V_{1}^{n_{j}} V_{2}^{-\left(Q_{j 2}+a m_{j}\right)} V_{3}^{p a l_{j}-k Q_{j 1}} \\
& +\xi_{j,+} H_{j,+}\left(V_{3}^{k}\right) V_{0}^{-m_{j}} V_{1}^{n_{j}} V_{2}^{-\left(Q_{j 2}+a m_{j}\right)+1} V_{3}^{p a l_{j}-k Q_{j 1}} \\
& +\xi_{j,-} H_{j,-}\left(V_{3}^{k}\right) V_{0}^{-m_{j}} V_{1}^{n_{j}} V_{2}^{-\left(Q_{j 2}+a m_{j}\right)-1} V_{3}^{p a l_{j}-k Q_{j 1}}
\end{aligned}
$$

where $\xi_{j 0}, \xi_{j, \pm} \in \mathbf{T}$ and

$$
\begin{aligned}
H_{j 0}(x)= & \sum_{n \in \mathbf{Z}} \sqrt{f_{0}(p \alpha(-x+n)) f_{0}\left(p \alpha(-x+n)+\left(\vec{v} \times \vec{\Theta}-\vec{Q}_{2}\right)_{j}\right)} \\
& \cdot \exp \left(2 \pi i(x+n)\left(\vec{Q}_{1}-\vec{\mu} \times \vec{\Theta}\right)_{j}\right), \quad j=1,2,3, \\
H_{j, \pm}(x)= & \sum_{n \in \mathbf{Z}} \sqrt{f_{0}(p \alpha(-x+n)) f_{0}\left(p \alpha(-x+n)+\left(\vec{v} \times \vec{\Theta}-\vec{Q}_{2}\right)_{j} \pm 1\right)} \\
& \cdot \exp \left(2 \pi i(x+n)\left(\vec{Q}_{1}-\vec{\mu} \times \vec{\Theta}\right)_{j}\right), \quad j=1,2,3 .
\end{aligned}
$$

Applying the estimate $(* 3)$ to these three functions, we deduce that

$$
e_{0} U_{j} e_{0} \sim \xi_{j 0} V_{0}^{-m_{j}} V_{1}^{n_{j}} V_{2}^{-\left(Q_{j 2}+a m_{j}\right)} V_{3}^{p a l_{j}-k Q_{j 1}}, \quad j=1,2,3,
$$

to within $8 / N+16 \sqrt{\varepsilon}+17 \pi \varepsilon$. 
Part Two. The $\left(1-e_{0}\right) A\left(1-e_{0}\right)$ part.

For the same group $G$ and the same vectors $\vec{m}, \vec{l}, \vec{n}$ as above, we define another lattice $\tilde{D}=\operatorname{span}\left(\tilde{\varepsilon}_{1}, \tilde{\varepsilon}_{2}, \tilde{\varepsilon}_{3}\right) \subseteq G$, where

$$
\left(\begin{array}{c}
\tilde{\varepsilon}_{1} \\
\tilde{\varepsilon}_{2} \\
\tilde{\varepsilon}_{3}
\end{array}\right)=\left(-\frac{1-p \alpha}{p} \vec{m}, \vec{n}, \vec{m}, \vec{l}, \frac{1}{k} \vec{l}, \vec{z}, s_{0} \vec{l}, 0\right) .
$$

The same argument as in Part One gives the identity $A=\mathrm{C}^{*}(\tilde{D}, \bar{\beta})$, where $U_{j}$ is identified with $\chi_{\tilde{\varepsilon}_{j}} /(1-p \alpha)$. Also, another argument as in Part One shows that $\tilde{D}^{\perp}=\operatorname{span}\left(\tilde{\delta}_{0}, \tilde{\delta}_{1}, \tilde{\delta}_{2}, \tilde{\delta}_{3}\right)$, where

$$
\left(\begin{array}{c}
\tilde{\delta}_{0} \\
\tilde{\delta}_{1} \\
\tilde{\delta}_{2} \\
\tilde{\delta}_{3}
\end{array}\right)=\left(\begin{array}{cccccccc}
0 & 0 & 0 & {[1]_{k}} & 0 & 0 & 0 & 0 \\
-k\langle\vec{m}, \vec{\Theta}\rangle & -1 & 0 & 0 & \frac{p}{1-p \alpha}\langle\vec{l}, \vec{\Theta}\rangle & 0 & 0 & 0 \\
0 & 0 & 0 & 0 & \frac{1}{1-p \alpha} & 0 & {[1]_{p}} & 0 \\
\frac{1}{p} & 0 & {[-1]_{p}} & 0 & 0 & 0 & 0 & {[a]_{k}}
\end{array}\right) .
$$

We denote $\chi_{\tilde{\delta}_{j}}$ by $\tilde{V}_{j}, j=1,2,3$.

If we take the Schwartz function $\tilde{f}$ on $\mathbf{R} \times \mathbf{Z} \times \mathbf{Z}_{p} \times \mathbf{Z}_{k}$ defined by

$$
\tilde{f}(x, 0,0,0)=\tilde{f}(-x, 0,0,0)=\sqrt{1-f_{0}(x)} \exp \frac{\pi i x}{1-p \alpha},
$$

for $0 \leqq x \leqq 1 / 2$ and zero otherwise, then a computation as in [EL1] shows that

$$
\frac{1}{1-p \alpha}\langle\tilde{f}, \tilde{f}\rangle_{\tilde{D}^{\perp}}=1_{\mathrm{C}^{*}\left(\tilde{D}^{\perp}, \beta\right)}, \quad \frac{1}{1-p \alpha}\langle\tilde{f}, \tilde{f}\rangle_{\tilde{D}}=1-e_{0}
$$

Thus we can identify $\left(1-e_{0}\right) A\left(1-e_{0}\right)$ with $\mathrm{C}^{*}\left(\tilde{D}^{\perp}, \beta\right)$ by the isomorphism

$$
\Phi_{2}:\left(1-e_{0}\right) c\left(1-e_{0}\right) \mapsto \frac{1}{1-p \alpha}\langle\tilde{f} * c, \tilde{f}\rangle_{\tilde{D}^{\perp}}
$$

Clearly, the four unitaries $\tilde{V}_{0}, \tilde{V}_{1}, \tilde{V}_{2}, \tilde{V}_{3}$ form standard generators of $\left(1-e_{0}\right) A\left(1-e_{0}\right)$.

For $j=1,2,3$ and $\varpi \in \tilde{D}^{\perp}$, we can calculate $(1 /(1-p \alpha))\left\langle\tilde{f} * U_{j}, \tilde{f}\right\rangle_{\tilde{D}^{\perp}}(\varpi)$ in a similar way as in Part One. This leads to

$$
\left(1-e_{0}\right) U_{j}\left(1-e_{0}\right) \sim \eta_{j} V_{0}^{-l_{j}} V_{1}^{n_{j}} V_{2}^{-\left(Q_{j 1}+l_{j}(1-p a) / k\right)} V_{3}^{m_{j}-p\left(Q_{j 2}+a m_{j}\right)},
$$

to within $8 / N+16 \sqrt{\varepsilon}+17 \pi \varepsilon$, where $\eta_{j}$ is in $\mathbf{T}$.

Notice that the inverse maps of $\Phi_{1}$ and $\Phi_{2}$ are given by

$$
\begin{aligned}
& \Phi_{1}^{-1}: c \mapsto \frac{1}{p \alpha}\langle f, c * f\rangle_{D}, \\
& \Phi_{2}^{-1}: \tilde{c} \mapsto \frac{1}{1-p \alpha}\langle\tilde{f}, \tilde{c} * \tilde{f}\rangle_{\tilde{D}}
\end{aligned}
$$

Using the continuous functional calculus, it is not hard to check that the unitaries $\Phi_{1}^{-1}\left(V_{j}\right)$ and $\Phi_{2}^{-1}\left(\tilde{V}_{j}\right)$ continuously vary as $N \rightarrow \infty$. By taking the limit, we pass the result from $e_{0}$ to $e$. 
Corollary. Under the same conditions as in the theorem, if in addition that

$$
\begin{aligned}
\operatorname{det}\left(\begin{array}{cc}
\left\langle\vec{\mu}, \vec{Q}_{2}\right\rangle & \left\langle\vec{v}, \vec{Q}_{2}\right\rangle \\
\left\langle\vec{\mu}, \vec{Q}_{1}\right\rangle & \left\langle\vec{v}, \vec{Q}_{1}\right\rangle
\end{array}\right) & =p a\left(p a+\left\langle\vec{\mu}, \vec{Q}_{2}\right\rangle-\left\langle\vec{v}, \vec{Q}_{1}\right\rangle\right) \\
& =(1-p a)\left(1-p a+\left\langle\vec{v}, \vec{Q}_{1}\right\rangle-\left\langle\vec{\mu}, \vec{Q}_{2}\right\rangle\right),
\end{aligned}
$$

then $\left\{e U_{j} e: j=1,2,3\right\}$ is approximately contained in a matrix algebra over an irrational rotation algebra (or direct sums of several copies) in eAe, to within $16 \sqrt{\varepsilon}+17 \pi \varepsilon$. So does $\left\{(1-e) U_{j}(1-e): j=1,2,3\right\}$ in $(1-e) A(1-e)$.

Proof. With the notations and the approximation to $U_{j}$ 's as in the above theorem, it is elementary to check that the condition $(* 4)$ is the same as

$$
\begin{aligned}
& \operatorname{det}\left(\vec{n},-\left(\vec{Q}_{2}+a \vec{m}\right), p a \vec{l}-k \vec{Q}_{1}\right) \\
& \quad=\operatorname{det}\left(\vec{n},-\left(\vec{Q}_{1}+\frac{(1-p a)}{k} \vec{l}\right), \vec{m}-p\left(\vec{Q}_{2}+a \vec{m}\right)\right)=0,
\end{aligned}
$$

which is equivalent to

and

$$
\operatorname{rank}\left\{\left[e U_{j} e\right]_{1}: j=1,2,3\right\}<3 \text { in } K_{1}(e A e)
$$

$$
\operatorname{rank}\left\{\left[(1-e) U_{j}(1-e)\right]_{1}: j=1,2,3\right\}<3 \quad \text { in } K_{1}((1-e) A(1-e)) .
$$

Let us consider the $e A e$ part, the consideration in the $(1-e) A(1-e)$ part is similar.

Since $\left[V_{1}\right]_{1},\left[V_{2}\right]_{1},\left[V_{3}\right]_{1}$ are integral independent in $K_{1}(e A e)$, there are two Laurent monomials $W_{1}, W_{2}$ of $V_{1}, V_{2}, V_{3}$, such that, for each $j=1,2,3$, $V_{1}^{n_{j}} V_{2}^{-\left(Q_{j 2}+a m_{j}\right)} V_{3}^{\text {pal }_{j}-k Q_{j 1}}$ is a Laurent monomial of $W_{1}$ and $W_{2}$, up to a scalar multiple.

Thus $\left\{e U_{j} e: j=1,2,3\right\}$ is approximately contained in $\mathrm{C}^{*}\left(V_{0}, W_{1}, W_{2}\right)$, to within $16 \sqrt{\varepsilon}+17 \pi \varepsilon$. We have to show that the $\mathrm{C}^{*}$-algebra $\mathrm{C}^{*}\left(V_{0}, W_{1}, W_{2}\right)$ has the expected form.

Since $A$ is simple, the embedding $e A e \mapsto A$ induces an ordered group isomorphism between their $K_{0}$-groups (Proposition 2.4 of [R1]). Thus by the uniqueness of the state of the ordered $K_{0}$-group with distinguished order unit, we have $(p \alpha) \cdot \tau_{e A e}\left(K_{0}(e A e)\right)=\tau_{A}\left(K_{0}(A)\right)$, where $\tau_{A}$ denotes the canonical trace on $A$. Consequently, if we denote by $\tilde{\theta}_{12}, \tilde{\theta}_{13}, \tilde{\theta}_{23}$ the commutation parameters of $V_{1}, V_{2}, V_{3}$, then $1, \tilde{\theta}_{12}, \tilde{\theta}_{13}, \tilde{\theta}_{23}$ are rationally independent. On the other hand, we must have

$$
\operatorname{rank}\left(\vec{n},-\left(\vec{Q}_{2}+a \vec{m}\right), p a \vec{l}-k \vec{Q}_{1}\right) \geqq 2 .
$$

Since otherwise, we would have

$$
\begin{aligned}
& \vec{Q}_{2}=-a \vec{m}+r_{1} \vec{n}, \\
& \vec{Q}_{1}=\frac{p a}{k} \vec{l}+r_{2} \vec{n},
\end{aligned}
$$

for some rational numbers $r_{1}, r_{2}$. Consequently,

$$
\left\langle\vec{v}, \vec{Q}_{1}\right\rangle=p a=-\left\langle\vec{\mu}, \vec{Q}_{2}\right\rangle .
$$

From $(* 4)$, we would reach a contradiction

$$
-(p a)^{2}=(1-p a)(1+p a)=1-(p a)^{2} .
$$


Thus the cross product of some two row vectors in the matrix $\left(\vec{n},-\left(\vec{Q}_{2}+a \vec{m}\right)\right.$, $\left.p a \vec{l}-k \vec{Q}_{1}\right)$ must be non-zero. The commutation relations in

$$
\left\{V_{1}^{n_{j}} V_{2}^{-\left(Q_{j 2}+a m_{j}\right)} V_{3}^{\text {pal }_{j}-k Q_{j 1}}: j=1,2,3\right\}
$$

cannot all be rational. Consequently, the commutation relation of $W_{1}$ and $W_{2}$ must be irrational. To show that the $\mathrm{C}^{*}$-algebra $\mathrm{C}^{*}\left(V_{0}, W_{1}, W_{2}\right)$ is isomorphic to some finite dimensional algebra tensored by an irrational rotation algebra, we notice that

$$
\left[V_{1}, V_{0}\right]=\left[V_{2}, V_{0}\right]=0 \text {. }
$$

We will denote by $\left(a_{1}, \ldots, a_{n}\right)$ the greatest common divisor of the integers $a_{1}, \ldots, a_{n}$. Writing

$$
W_{1}=V_{1}^{a_{1}} V_{2}^{b_{1}} V_{3}^{c_{1}} \text { and } W_{2}=V_{1}^{a_{2}} V_{2}^{b_{2}} V_{3}^{c_{2}},
$$

we have the following three cases only:

(1) If $c_{1} \equiv c_{2} \equiv 0(\bmod p)$, then

$$
\mathrm{C}^{*}\left(V_{0}, W_{1}, W_{2}\right) \cong \mathbf{C}^{p} \otimes \mathrm{C}^{*}\left(W_{1}, W_{2}\right) .
$$

(2) If we have only one $c_{j} \equiv 0(\bmod p)$, say $j=1$, then a slight modification of the argument in [EL1] (Lemma 2 and the proof at the end of Part One) gives that

$$
\mathrm{C}^{*}\left(V_{0}, W_{1}, W_{2}\right) \cong 1_{\left(c_{2}, p\right)} \otimes M_{p /\left(c_{2}, p\right)} \otimes \mathrm{C}^{*}\left(W_{1}, W_{2}^{p /\left(c_{2}, p\right)}\right) .
$$

(3) If both $c_{1}$ and $c_{2}$ are not integral multiples of $p$, then there is a pair of integers $u$ and $v$ such that

$$
\left(c_{1}, c_{2}\right)=c_{1} u+c_{2} v \text {. }
$$

Then a change of coordinate in $\mathrm{C}^{*}\left(W_{1}, W_{2}\right)$ gives that

$$
\begin{gathered}
\tilde{W}_{1}=W_{1}^{c_{2} /\left(c_{1}, c_{2}\right)} W_{2}^{-c_{1} /\left(c_{1}, c_{2}\right)}=\eta V_{1}^{\left(a_{1} c_{2}-a_{2} c_{1}\right) /\left(c_{1}, c_{2}\right)} V_{2}^{\left(b_{1} c_{2}-b_{2} c_{1}\right) /\left(c_{1}, c_{2}\right)}, \\
\tilde{W}_{2}=W_{1}^{u} W_{2}^{v}=\tilde{\eta} V_{1}^{a_{1} u+a_{2} v} V_{2}^{b_{1} u+b_{2} v} V_{3}^{\left(c_{1}, c_{2}\right)},
\end{gathered}
$$

where $\eta, \tilde{\eta} \in \mathbf{T}$. We have that

$$
\left[\tilde{W}_{1}, V_{0}\right]=0 \quad \text { and } \quad V_{0} \tilde{W}_{2}=\tilde{W}_{2} V_{0} \exp \left(2 \pi i \frac{s_{0}\left(c_{1}, c_{2}\right)}{p}\right) .
$$

A similar argument as above gives that

$$
\mathrm{C}^{*}\left(V_{0}, W_{1}, W_{2}\right) \cong 1_{\left(c_{1}, c_{2}, p\right)} \otimes M_{p /\left(c_{1}, c_{2}, p\right)} \otimes \mathrm{C}^{*}\left(\tilde{W}_{1}, \tilde{W}_{2}^{p /\left(c_{1}, c_{2}, p\right)}\right) .
$$

In any case, we have the desired form for $\mathrm{C}^{*}\left(V_{0}, W_{1}, W_{2}\right)$. This completes the proof.

\section{Remark.}

(1) The converse of this corollary is clearly true by considering the rank of certain $K_{1}$-classes. But we will not need it in this paper.

(2) The above corollary combining the theorem of Elliott and Evans ([EE]) gives an approximation to $\left\{U_{1}, U_{2}, U_{3}\right\}$ by direct sums of four circle algebras in $A$, 
provided by the existence of a pair of integral vectors $(\vec{\mu}, \vec{v})$ which satisfies the above conditions.

\section{The Main Theorem}

In this section, a non-commutative 3-torus $A$ may not be simple.

The Main Theorem. If $\left(1, \theta_{12}, \theta_{13}, \theta_{23}\right)$ are rationally independent, then the corresponding non-commutative 3-torus is an inductive limit of direct sums of four circle algebras.

Assuming this theorem, we shall first prove the following corollaries.

Corollary 1. Any simple non-commutative 3-torus is an inductive limit of four circle algebras. Therefore, they are classified by their ordered $K_{0}$-group with distinguished order unit.

Proof. The first statement is a combination of the main theorem here and the main theorem in [EL2]. Once we have the first statement, the second statement follows easily from the classification theorem of Elliott (Theorem 7.1 of [E]).

Corollary 2. (1) Any non-commutative 3-torus is an inductive limit of type I $\mathrm{C}^{*}$-algebras. More precisely, it is either an inductive limit of direct sums of four circle algebras, or an inductive limit of direct sums of two $\mathrm{C}^{*}$-algebras each of which is a matrix algebra over a rational rotation algebra (including the commutative 2-torus), or a rational 3-torus.

(2) Two 3-tori are stably isomorphic if and only if the range of the canonical trace on the $K_{0}$-group of one torus is a real multiple of that of another torus.

Proof. Following from some standard fact and Proposition 1 in [EL2], we know that a non-commutative 3 -torus $A$ is non-simple if and only if

$$
\operatorname{rank}\left(\tau_{A}\left(K_{0}(A)\right)\right) \leqq 2,
$$

and rational if and only if

$$
\operatorname{rank}\left(\tau_{A}\left(K_{0}(A)\right)\right)=1,
$$

where $\tau_{A}$ denotes the canonical trace on $A$.

Thus the first statement is obvious in the case where

$$
\operatorname{rank}\left(\tau_{A}\left(K_{0}(A)\right)\right) \neq 2 .
$$

Now, we consider the case where $A$ is a 3 -torus with

$$
\operatorname{rank}\left(\tau_{A}\left(K_{0}(A)\right)\right)=2 .
$$

In this case, an easy consequence of a theorem of Poguntke ([Po], see the appendix of our paper for a direct proof) asserts that $A$ is strongly Morita equivalent to $A_{\theta} \otimes C(\mathbf{T})$, where $\theta$ is an irrational number.

Applying the main theorem of [EE], in this situation, there is an increasing sequence of unital $C^{*}$-subalgebras $\left\{A_{n}\right\}$ in some unital $C^{*}$-algebra such that

$$
A_{n} \cong M_{q_{n}}\left(C\left(\mathbf{T}^{2}\right)\right) \oplus M_{q_{n}^{\prime}}\left(C\left(\mathbf{T}^{2}\right)\right), \quad n \geqq 1 ;
$$


and there is a projection $e \in \overline{\bigcup_{n=1}^{\infty} A_{n}}$ such that

$$
e \bigcup_{n=1}^{\infty} A_{n} e \cong A .
$$

For a sufficiently large $n$, there is a projection $e^{\prime}$ in $A_{n}$ and a unitary $W$ in $\overline{\bigcup_{n=1}^{\infty} A_{n}}$ such that

$$
\operatorname{Ad}(W)\left(e^{\prime}\right)=e
$$

Notice that

$$
\operatorname{Ad}(W)\left(A_{n}\right) \cong A_{n} \cong M_{q_{n}}\left(C\left(\mathbf{T}^{2}\right)\right) \oplus M_{q_{n}^{\prime}}\left(C\left(\mathbf{T}^{2}\right)\right)
$$

We may replace the sequence $\left\{A_{n}\right\}$ by $\left\{\operatorname{Ad}(W)\left(A_{n}\right)\right\}$ and therefore $e$ is in $\operatorname{Ad}(W)\left(A_{n}\right)$, for all sufficiently large $n$.

Then the first statement in this case follows from the bijective correspondence between the isomorphic classes of the algebras, each of which is a matrix algebra over a rational rotation algebra, and the equivalent classes of projections in $C\left(\mathbf{T}^{2}\right) \otimes K$ (cf. Sect. 3 in [R2]).

For the second statement, the "only if" part follows easily from the following general fact (we will supply a proof in the appendix): for two $n$-tori $A$ and $B$,

$$
\begin{gathered}
\qquad\left(K_{0}(A), K_{0}^{+}(A)\right) \cong\left(K_{0}(B), K_{0}^{+}(B)\right) \\
\Leftrightarrow \text { there is } \gamma \in \mathbf{R}^{+} \text {such that } \gamma \cdot \tau_{A}\left(K_{0}(A)\right)=\tau_{B}\left(K_{0}(B)\right) .
\end{gathered}
$$

Now, for two 3-tori $A$ and $B$ satisfying the condition of the "if" part of the second statement of the corollary, let us consider the argument used in the beginning of the proof of this corollary. We can see that $A$ and $B$ are either both simple or both non-simple, both rational or both non-rational. Thus the "if" part of the second statement follows from the classification theorem of Elliott for simple tori (Theorem 7.1 of $[\mathrm{E}]$ ) and the Morita equivalence result of Rieffel for rational tori (Theorem 3.1 of [R2]).

To finish the proof, assume that $A$ and $B$ are two 3-tori satisfying the condition $(* 5)$ and the additional condition that

$$
\operatorname{rank}\left(\tau_{A}\left(K_{0}(A)\right)\right)=\operatorname{rank}\left(\tau_{B}\left(K_{0}(B)\right)\right)=2
$$

Then by a consequence of Poguntke's theorem (see [Po] and the appendix of our paper), we have

$$
A \otimes K \cong A_{\theta_{1}} \otimes C(\mathbf{T}) \otimes K \quad \text { and } \quad B \otimes K \cong A_{\theta_{2}} \otimes C(\mathbf{T}) \otimes K,
$$

where $\theta_{1}$ and $\theta_{2}$ are two irrational numbers.

Following from our proof of this result in the appendix, we can choose $\theta_{1}$ and $\theta_{2}$ in the way that there are positive integers $q_{1}$ and $q_{2}$ such that $\left\{1 / q_{1}, \theta_{1} / q_{1}\right\}$ forms an integral basis of $\tau_{A}\left(K_{0}(A)\right)$ and $\left\{1 / q_{2}, \theta_{2} / q_{2}\right\}$ forms a basis of $\tau_{B}\left(K_{0}(B)\right)$.

Since

$$
\gamma \cdot \tau_{A}\left(K_{0}(A)\right)=\tau_{B}\left(K_{0}(B)\right)
$$


$\left\{\gamma / q_{1}, \gamma \theta_{1} / q_{1}\right\}$ is an integral basis of $\tau_{B}\left(K_{0}(B)\right)$ as well. Therefore, there is a matrix $S$ in $G L(2, \mathbf{Z})$ such that

$$
S\left(1, \theta_{2}\right)^{\top}=\gamma^{\prime}\left(1, \theta_{1}\right)^{\top},
$$

where $\gamma^{\prime}=\gamma q_{2} / q_{1}$. This shows that (see Theorem 4 of [R1])

$$
A_{\theta_{1}} \otimes K \cong A_{\theta_{2}} \otimes K \text {. }
$$

Thus $A$ and $B$ are stably isomorphic.

Because of the corollary of the local reduction theorem and the remark afterward, to prove the main theorem, we only need to find pairs of non-zero integral vectors $(\vec{\mu}, \vec{v})$ satisfying all the conditions in the local reduction theorem as well as those in its corollary.

In the article [EL2], we proved a number theoretical lemma, which will serve as our starting point.

A Number Theoretical Lemma. For each vector $\vec{\theta}=\left(1, \theta_{1}, \ldots, \theta_{n}\right)$ with rationally independent components (where $0<\theta_{j}<1$ ) and $0<\varepsilon<1 /(n+2)$, there is a positive integral basis $\vec{A}_{1}, \ldots, \vec{A}_{n+1}$, such that

(1) the vector $\vec{\theta}$ is in the positive cone generated by $\vec{A}_{j}^{\prime}$ 's, namely,

(2) $\operatorname{dist}\left(\vec{A}_{j}, \vec{\theta} \mathbf{R}\right)<\varepsilon$;

$$
\vec{\theta}=\sum_{j=1}^{n+1} \alpha_{j} \vec{A}_{j}, \quad \alpha_{j}>0
$$

(3) if $p_{1 j}$ denotes the first component of $\vec{A}_{j}$, then there is a $1 \leqq j \leqq n+1$ such that

$$
p_{1 j}>\frac{1}{\varepsilon} \text { and } \frac{1}{(n+2) n}<p_{1 j} \alpha_{j}<\frac{1}{2} \text {. }
$$

Notice that, when $n=3$, the condition that

$$
\frac{1}{15}<p_{1 j} \alpha_{j}<\frac{1}{2}
$$

implies that there are $1 \leqq k, i \leqq 4$ with $k \neq i$ such that

$$
\frac{1}{15}<p_{1 i} \alpha_{i}+p_{1 k} \alpha_{k}<\frac{1}{2} \text {. }
$$

To see this, let us observe that there exists a $i \neq j$ such that

We have either

$$
p_{1 i} \alpha_{i}<\frac{1}{3} \text {. }
$$

$$
\frac{1}{15}<p_{1 i} \alpha_{i}+p_{1 j} \alpha_{j}<\frac{1}{2},
$$

or for $l \neq i, j$ and $k \neq i, j$,

$$
\frac{1}{6}<\sum_{q \neq j} p_{1 q} \alpha_{q}-\frac{1}{3}<p_{1 k} \alpha_{k}+p_{1 l} \alpha_{l}<\frac{1}{2} .
$$

In either case, we have $(* 6)$ inequality. 
Thus if we take $\vec{\theta}=\left(1, \theta_{12}, \theta_{13}, \theta_{23}\right)$ and an integral basis $\left\{\vec{A}_{1}, \vec{A}_{2}, \vec{A}_{3}, \vec{A}_{4}\right\}$ satisfying the corresponding conditions in the number theoretical lemma above, then we may assume

$$
\frac{1}{15}<p_{11} \alpha_{1}+p_{12} \alpha_{2}<\frac{1}{2} .
$$

Also, we denote by $\left(p_{i j}\right)$ the matrix $\left(\vec{A}_{1}, \vec{A}_{2}, \vec{A}_{3}, \vec{A}_{4}\right)$, and we may assume $\operatorname{det}\left(p_{i j}\right)=1$.

Lemma 2. Denote by $\vec{P}_{j}$ the vector $\left(p_{2 j}, p_{3 j}, p_{4 j}\right)^{\top}$ in $\mathbf{Z}^{3}$ and by $\left(a_{i j}\right)$ the inverse matrix of $\left(p_{i j}\right)$. Then

$$
p_{11} \alpha_{1}+p_{12} \alpha_{2}=p_{11} a_{11}+p_{12} a_{21}+\left\langle\left(p_{11} \vec{P}_{2}-p_{12} \vec{P}_{1}\right) \times\left(p_{13} \vec{P}_{4}-p_{14} \vec{P}_{3}\right), \vec{\Theta}\right\rangle,
$$

where $\vec{\Theta}=\left(\theta_{12}, \theta_{13}, \theta_{23}\right)$. (Warning: Do not confuse this with " $\vec{\Theta}$ " used in the last section!)

Proof. From the definition of $\alpha_{j}$ 's, we know that

$$
\alpha_{j}=a_{j 1}+a_{j 2} \theta_{12}+a_{j 3} \theta_{13}+a_{j 4} \theta_{23} .
$$

Thus it suffices to prove that

$$
p_{11}\left(\begin{array}{l}
a_{12} \\
a_{13} \\
a_{14}
\end{array}\right)+p_{12}\left(\begin{array}{l}
a_{22} \\
a_{23} \\
a_{24}
\end{array}\right)=\left(p_{11} \vec{P}_{2}-p_{12} \vec{P}_{1}\right) \times\left(p_{13} \vec{P}_{4}-p_{14} \vec{P}_{3}\right) .
$$

But this follows directly from the general algebraic relation of the $a_{i j}$ 's and the $p_{i j}$ 's.

Denote by $\vec{\mu}$ the vector $p_{11} \vec{P}_{2}-p_{12} \vec{P}_{1}$ and by $\vec{v}$ the vector $p_{13} \vec{P}_{4}-p_{14} \vec{P}_{3}$. We will see that the pair $(\vec{\mu}, \vec{v})$ satisfies all the conditions required for the local reduction theorem as well as its corollary.

Lemma 3. The integral vector

is primitive.

$$
\frac{\vec{\mu}}{\left(p_{11}, p_{12}\right)} \times \frac{\vec{v}}{\left(p_{13}, p_{14}\right)}
$$

Proof. Since, for any integral vector $X,\langle\vec{\mu} \times \vec{v}, X\rangle$ is a multiple of $\left(p_{11}, p_{12}\right)$ $\left(p_{13}, p_{14}\right)$, it suffices to show that

$$
\left(p_{11}, p_{12}\right)\left(p_{13}, p_{14}\right) \in\left\langle\vec{\mu} \times \vec{v}, \mathbf{Z}^{3}\right\rangle .
$$

Observe that

$$
\begin{aligned}
& \left\langle\vec{\mu} \times \vec{v}, \vec{P}_{1}\right\rangle=p_{11}\left(p_{13} a_{31}+p_{14} a_{41}\right), \\
& \left\langle\vec{\mu} \times \vec{v}, \vec{P}_{2}\right\rangle=p_{12}\left(p_{13} a_{31}+p_{14} a_{41}\right), \\
& \left\langle\vec{\mu} \times \vec{v}, \vec{P}_{3}\right\rangle=p_{13}\left(p_{11} a_{11}+p_{12} a_{21}\right), \\
& \left\langle\vec{\mu} \times \vec{v}, \vec{P}_{4}\right\rangle=p_{14}\left(p_{11} a_{11}+p_{12} a_{21}\right) .
\end{aligned}
$$


Thus $\left(p_{11}, p_{12}\right)\left(p_{13} a_{31}+p_{14} a_{41}\right)$ and $\left(p_{13}, p_{14}\right)\left(p_{11} a_{11}+p_{12} a_{21}\right)$ are both in $\left\langle\vec{\mu} \times \vec{v}, \mathbf{Z}^{3}\right\rangle$. The expected conclusion follows from the identity

$$
p_{11} a_{11}+p_{12} a_{21}+p_{13} a_{31}+p_{14} a_{41}=1 .
$$

Notice that the above proof also shows the rational independence of $\vec{\mu}$ and $\vec{v}$.

Proof of the Main Theorem. Using the notations as in the technical condition $(* 2)$, Lemma 3 tells us that, for our special choice of $(\vec{\mu}, \vec{v})$ from Lemma 2,

$$
p=\left(p_{11}, p_{12}\right), \quad k=\left(p_{13}, p_{14}\right) \quad \text { and } \quad p a=p_{11} a_{11}+p_{12} a_{21} \text {. }
$$

Therefore, the technical condition $(* 2)$ is satisfied by this pair $(\vec{\mu}, \vec{v})$.

Notice that

$$
\left\|\vec{P}_{j}-p_{1 j} \vec{\Theta}\right\| \leqq \operatorname{dist}\left(\vec{A}_{j}, \vec{\theta} \mathbf{R}\right)<\varepsilon, \quad j=1,2,3,4,
$$

the extra conditions in the local reduction theorem follow from the following two identities:

$$
\begin{aligned}
& \vec{\mu} \times \vec{\Theta}+\vec{P}_{1} \times \vec{P}_{2}=\left(\vec{P}_{1}-p_{11} \vec{\Theta}\right) \times\left(\vec{P}_{2}-p_{12} \vec{\Theta}\right) \\
& \vec{v} \times \vec{\Theta}+\vec{P}_{3} \times \vec{P}_{4}=\left(\vec{P}_{3}-p_{13} \vec{\Theta}\right) \times\left(\vec{P}_{4}-p_{14} \vec{\Theta}\right)
\end{aligned}
$$

Finally, it is easy to check that the conditions $(* 4)$ in the corollary of the local reduction theorem are all satisfied. Thus by this corollary, the cut-down of $\left\{U_{1}, U_{2}, U_{3}\right\}$ by the projection $e\left(U^{\vec{v}}, U^{\vec{\mu}}\right)$ will lead to the desired local approximation by direct sums of circle algebras.

We make a few remarks.

\section{Remarks.}

(1) The work above and the work of [EL2] together show that, for each simple 3-torus $A$ with a fixed canonical unitary generator $\left\{U_{1}, U_{2}, U_{3}\right\}$ and each $\varepsilon>0$, there is a unital $\mathrm{C}^{*}$-subalgebra $A_{\varepsilon}$ of $A$ with the same unit such that

(i) $A_{\varepsilon} \cong$ direct sums of four circle algebras,

(ii) the unitary $U_{1}, U_{2}, U_{3}$ are all within the distance $\varepsilon$ from $A_{\varepsilon}$.

Then, by a general procedure as described in [Br], we can express $A$ as an inductive limit of direct sums of four circle algebras. We briefly describe the induction step of this procedure to remind the reader.

Suppose we have $1 \in A_{n_{k}} \subset A$ and a finite subset $Y_{k} \subset A_{n_{k}}$, where $A_{n_{k}}$ is a unital copy of direct sums of four circle algebras. Let us fix canonical generators $a_{i}$ 's of $A_{n_{k}}$.

For each $\delta_{k}$, there is a $\mathrm{C}^{*}$-subalgebra $A_{n_{k+1}}$ of $A$ containing $1_{A}$ such that $U_{1}, U_{2}, U_{3}$ are all within the distance $\frac{\delta_{k}}{2^{k+1}}$ from $A_{n_{k+1}}$ and, for each $i$,

$$
a_{i} \sim \tilde{a}_{i} \in A_{n_{k+1}}
$$

to within $\frac{\delta_{k}}{2^{k+1}}$, where $A_{n_{k+1}}$ is a unital copy of direct sums of four circle algebras.

Then by the stability of the generating relation of circle algebras (cf. [E]), we can perturb the set $\left\{\tilde{a}_{i}\right\}$ to a set $\left\{\hat{a}_{i}\right\} \subset A_{n_{k+1}}$ such that

(i) $a_{i} \sim \hat{a}_{i}$, to within $\frac{f\left(\delta_{k}\right)}{2^{k+1}}$, for each $i$, where $f$ is a positive function depending on the algebraic type of $A_{n_{k}}$ only and $f(t) \rightarrow 0$ when $t \rightarrow 0$, 
(ii) the assignment $\left\{a_{i} \mapsto \hat{a}_{i}: i\right\}$ can be extended to a $\mathrm{C}^{*}$-algebraic embedding of $A_{n_{k}}$ into $A_{n_{k+1}}$. We denote it by $\phi_{k}$.

Now, we can choose $\delta_{k}$ so small that the diagram

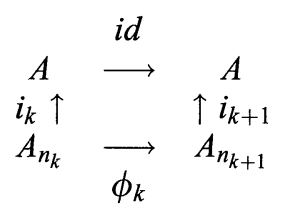

approximately commutes on the set $\left\{a_{i}\right\} \cup Y_{k}$ to within $\frac{1}{2^{k+1}}$, where $i_{k}$ is the natural inclusion map. We choose a finite subset $Y_{k+1} \subset A_{n_{k+1}}$ such that

$$
\phi_{k}\left(Y_{k} \cup\left\{a_{i}\right\}\right) \subset Y_{k+1} \text {. }
$$

Finally, an argument similar to the approximately intertwining one as described in the beginning of [E] shows that, with $Y_{k}$ chosen inductively and carefully,

$$
\lim _{\rightarrow k} i_{k}: \lim _{\rightarrow k}\left(A_{n_{k}}, \phi_{k}\right) \mapsto A
$$

is a $\mathrm{C}^{*}$-algebraic isomorphism.

(2) In the same way as in [Bo], one can show that the flip fixed point algebra of any simple 3-torus is AF-algebra.

(3) The success in our case might indicate some general way to show the quasidiagonality of the crossed product of certain non-commutative $C^{*}$-algebras by $\mathbf{Z}$.

(4) The method used here might be possible to extend to a higher dimensional simple torus. At first, the local reduction theorem and its corollary can be extended to higher dimensional tori without essential difficulty. The difficulty lies in the Diophantine approximation. It seems that we should understand how the equivalence classes of projections with different trace order (i.e. the order of polynomial in $\theta_{i j}$ 's) sitting inside the order structure of the $K_{0}$-group. Are they really indistinguishable from the ordered group structure?

\section{Appendix}

We first give a direct proof of a consequence of Poguntke's theorem ([Po]) by some standard constructions of rotation algebras.

Recall that a projection in a $\mathrm{C}^{*}$-algebra is full if the closed 2-sided ideal generated by this projection is the entire $\mathrm{C}^{*}$-algebra.

Lemma 4. Every non-zero projection of a rational rotation algebra is full.

Proof. Let us look at $A_{p / q}$, where $p$ and $q$ are positive integers which are relatively prime. It is well known that we can view $A_{p / q}$ as a $M_{q}(\mathbf{C})$-bundle over $\mathbf{T}^{2}$. Let $e$ be a non-zero projection of $A_{p / q}$ and denote by $\langle e\rangle$ the closed 2-sided ideal generated by $e$. Since $T^{2}$ is connected, $e(x) \neq 0$ for each $x \in T^{2}$. Since $M_{q}(\mathbf{C})$ is simple, at each point $x$ in $\mathbf{T}^{2}$, there is a positive element $a_{x}$ in $\langle e\rangle$ such that $a_{x}(x)$ is invertible in $M_{q}(\mathbf{C})$. Combining the compactness of $\mathbf{T}^{2}$ and the elementary fact that, for any two $q \times q$ positive matrices $A$ and $B$ with one of them invertible, the matrix $A+B$ is invertible, we get an invertible element in $\langle e\rangle$. This leads to the conclusion. 
Proposition 5. Any non-commutative 3-torus is stably isomorphic to its center tensored by a simple torus.

Proof. Let $A$ be a non-simple 3-torus with commutation parameters $\theta_{i j}$ 's. By Proposition 1 of [EL2], we know that

$$
\operatorname{rank}\left(\tau_{A}\left(K_{0}(A)\right)\right) \leqq 2,
$$

where $\tau_{A}$ is the canonical trace on $A$. If

$$
\operatorname{rank}\left(\tau_{A}\left(K_{0}(A)\right)\right)=1,
$$

then $A$ must be a rational torus which is stably isomorphic to its center (cf. [R2]). So we may assume that

$$
\operatorname{rank}\left(\tau_{A}\left(K_{0}(A)\right)\right)=2 \text {. }
$$

We claim that there is a $\theta^{\prime} \in \tau_{A}\left(K_{0}(A)\right)$ and an integer $q$ such that

$$
\frac{1}{q} \mathbf{Z}+\theta^{\prime} \mathbf{Z}=\tau_{A}\left(K_{0}(A)\right) \text {. }
$$

To see this, let $\theta_{1}, \theta_{2}$ be any basis of the rank 2 free Abelian group $\tau_{A}\left(K_{0}(A)\right)$. We may assume that none of $\theta_{j}$ 's is of the form $1 / q$ for some integer $q$. Thus, there are two nonzero integers $a$ and $b$ such that

$$
1=a \theta_{1}+b \theta_{2} \text {. }
$$

Denote by $q$ the g.c.d. $(a, b)$. There are integers $u$ and $v$ such that

Consequently,

$$
\frac{a}{q} u+\frac{b}{q} v=1
$$

$$
\begin{aligned}
& \theta_{1}=\frac{u}{q}+\frac{b}{q}\left(v \theta_{1}-u \theta_{2}\right) \\
& \theta_{2}=\frac{v}{q}-\frac{a}{q}\left(v \theta_{1}-u \theta_{2}\right)
\end{aligned}
$$

Then, we may take $\theta^{\prime}=v \theta_{1}-u \theta_{2}$ and write

$$
\Theta:=\left(\begin{array}{ccc}
0 & \theta_{12} & \theta_{13} \\
-\theta_{12} & 0 & \theta_{23} \\
-\theta_{13} & -\theta_{23} & 0
\end{array}\right)=\frac{1}{q} C+\left(v \theta_{1}-u \theta_{2}\right) \tilde{C},
$$

where $C$ and $\tilde{C}$ are integral skew symmetric matrices.

Applying Theorem IV.1. of [N] to $\tilde{C}$, up to an integral conjugation, we may assume that

$$
\Theta=\left(\begin{array}{ccc}
0 & \theta & 0 \\
-\theta & 0 & \frac{p^{\prime}}{q^{\prime}} \\
0 & -\frac{p^{\prime}}{q^{\prime}} & 0
\end{array}\right),
$$

where $\theta$ is an irrational number and $q^{\prime}, p^{\prime}$ are integers with $\left(p^{\prime}, q^{\prime}\right)=1$. 
Let $U_{1}, U_{2}, U_{3}$ be unitary generators of $A$ with $(* 7)$ as the matrix of their commutation parameters, then

$$
\mathrm{C}^{*}\left(U_{2}, U_{3}\right) \cong A_{p^{\prime} / q^{\prime}}
$$

Let us take a minimal projection $e$ of $\mathrm{C}^{*}\left(U_{2}, U_{3}\right)$, which is full in $\mathrm{C}^{*}\left(U_{2}, U_{3}\right)$ (by Lemma 4 above), and a unitary $W$ in $\mathrm{C}^{*}\left(U_{2}, U_{3}\right)$ such that

$$
\operatorname{Ad}\left(U_{1}\right)(e)=\operatorname{Ad}(W)(e) .
$$

At first, following the strong Morita equivalence of a rational rotation algebra and the commutative 2-torus (cf. Sect. 3 of [R2]), we have

$$
e \mathrm{C}^{*}\left(U_{2}, U_{3}\right) e=\mathrm{C}^{*}\left(e U_{2}^{q^{\prime}}, e U_{3}^{q^{\prime}}\right) .
$$

Secondly, since $U_{1}^{k} W U_{1}^{-k} \in \mathrm{C}^{*}\left(U_{2}, U_{3}\right)$, by induction, we have that

$$
U_{2}^{n_{2}} U_{3}^{n_{3}} U_{1}^{n_{1}} \in \mathrm{C}^{*}\left(U_{2}, U_{3}\right) \cdot\left(W^{*} U_{1}\right)^{n_{1}}
$$

For instance, one can check

Therefore,

$$
U_{2}^{n_{2}} U_{3}^{n_{3}} U_{1}^{2}=U_{2}^{n_{2}} U_{3}^{n_{3}}\left(U_{1} W U_{1}^{*}\right) W\left(W^{*} U_{1}\right)^{2} .
$$

$$
\begin{aligned}
e \mathrm{C}^{*}\left(U_{1}, U_{2}, U_{3}\right) e & =e \mathrm{C}^{*}\left(W^{*} U_{1}, U_{2}, U_{3}\right) e=\mathrm{C}^{*}\left(e W^{*} U_{1}, e U_{2}^{q^{\prime}}, e U_{3}^{q^{\prime}}\right) \\
& \cong \mathrm{C}^{*}\left(e U_{3}^{q^{\prime}}\right) \otimes \mathrm{C}^{*}\left(e W^{*} U_{1}, e U_{2}^{q^{\prime}}\right) \cong C(\mathbf{T}) \otimes A_{q^{\prime} \theta}
\end{aligned}
$$

where we use Ex. 2 in p. 220 of [T] to get the tensor product expression.

Notice that the projection $e$ is full in $\mathrm{C}^{*}\left(U_{1}, U_{2}, U_{3}\right)$ as well. The rest follows from a result of [BGR] concerning Morita equivalence and stable isomorphism.

Proposition 6. Let $A$ and $B$ be two non-commutative $n$-tori, then

$$
\left(K_{0}(A), K_{0}^{+}(A)\right) \cong\left(K_{0}(B), K_{0}^{+}(B)\right)
$$

$\Longleftrightarrow$ there is a positive real number $\gamma$ such that $\gamma \cdot \tau_{A}\left(K_{0}(A)\right)=\tau_{B}\left(K_{0}(B)\right)$.

Proof. Since a rational $n$-torus $A$ is strongly Morita equivalent to $C\left(\mathbf{T}^{n}\right)$ (cf. [R2]), in this case we have that

$$
\left(K_{0}(A), K_{0}^{+}(A)\right) \cong\left(K_{0}\left(C\left(\mathbf{T}^{n}\right), K_{0}^{+}\left(C\left(\mathbf{T}^{n}\right)\right)\right) .\right.
$$

Therefore by the uniqueness of the state on $\left(K_{0}\left(C\left(\mathbf{T}^{n}\right), K_{0}^{+}\left(C\left(\mathbf{T}^{n}\right)\right),\left[1_{C\left(\mathbf{T}^{n}\right)}\right]\right)\right.$ (see 6.10.3. of [B]), any non-zero ordered group map from $\left(K_{0}(A), K_{0}^{+}(A)\right)$ into $\mathbf{R}$ is a multiple of $\tau_{A}$ and $\operatorname{rank}\left(\tau_{A}\left(K_{0}(A)\right)\right)=1$. As an easy consequence, if $A$ is rational, the following four statements are equivalent:

(1) $\left(K_{0}(A), K_{0}^{+}(A)\right) \cong\left(K_{0}(B), K_{0}^{+}(B)\right)$;

(2) $\operatorname{rank}\left(\tau_{A}\left(K_{0}(A)\right)\right)=\operatorname{rank}\left(\tau_{B}\left(K_{0}(B)\right)\right)=1$;

(3) there is a real number $\gamma$ such that $\gamma \cdot \tau_{A}\left(K_{0}(A)\right)=\tau_{B}\left(K_{0}(B)\right)$;

(4) $B$ is rational.

Therefore we only need to consider the case where $A$ and $B$ are both nonrational. We will keep this convention for the rest of the proof. 
Following from Rieffel ([R3]), the order structure for the $K_{0}$-group of a nonrational torus is the strict one induced from the canonical trace.

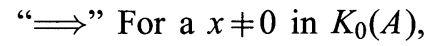

$$
\tau_{A}(x)=0 \quad \text { if and only if for any non-zero } y \in K_{0}^{+}(A), \tau_{A}(y \pm x)>0 .
$$

The "only if" part of this statement is trivial. To see the converse, suppose that $\tau_{A}(x)>0$ (consider $-x$ if $\tau_{A}(x)<0$ ), then $x>0$ and $\tau_{A}(x-x)=0$. Thus

Let $\varphi:\left(K_{0}(A), K_{0}^{+}(A)\right) \longmapsto\left(K_{0}(B), K_{0}^{+}(B)\right)$ be an ordered group isomorphism.

$$
\begin{gathered}
\tau_{A}(x)=0 \Longleftrightarrow \text { for any } y>0 \text { in } K_{0}(A), \quad y \pm x>0, \\
\Longleftrightarrow \text { for any } z>0 \text { in } K_{0}(B), \quad z \pm \varphi(x)>0, \\
\Longleftrightarrow \tau_{B}(\varphi(x))=0 .
\end{gathered}
$$

Consequently, $\varphi$ induces an ordered group isomorphism $\tilde{\varphi}$ from $\tau_{A}\left(K_{0}(A)\right)$ onto $\tau_{B}\left(K_{0}(B)\right)$ such that

$$
\tilde{\varphi} \circ \tau_{B}=\tau_{A} \circ \varphi .
$$

Since both $A$ and $B$ are non-rational, the additive subgroups $\tau_{A}\left(K_{0}(A)\right)$ and $\tau_{B}\left(K_{0}(B)\right)$ of $\mathbf{R}$ are both dense in $\mathbf{R}$. Then a standard argument shows that the ordered isomorphism $\tilde{\varphi}$ maps Cauchy sequence to Cauchy sequence and vice versa. Therefore, the map $\tilde{\varphi}$ extends uniquely to an additive automorphism of $\mathbf{R}$ by continuity. A well-known elementary argument shows that $\tilde{\varphi}$ must be linear. The conclusion follows.

"£" Define an ordered group isomorphism of $\tau_{A}\left(K_{0}(A)\right)$ onto $\tau_{B}\left(K_{0}(B)\right)$ by

$$
\tilde{\varphi}(x)=\gamma x,
$$

where $\gamma>0$ is the one appeared in the statement of the proposition.

Since $\tau_{A}\left(K_{0}(A)\right)$ and $\tau_{B}\left(K_{0}(B)\right)$ are free,

$$
\begin{aligned}
& K_{0}(A) \cong \tau_{A}\left(K_{0}(A)\right) \oplus \operatorname{ker} \tau_{A}, \\
& K_{0}(B) \cong \tau_{B}\left(K_{0}(B)\right) \oplus \operatorname{ker} \tau_{B} .
\end{aligned}
$$

Since $K_{0}(A)$ and $K_{0}(B)$ are both free Abelian groups of the same rank, the group isomorphism $\tilde{\varphi}$ of $\tau_{A}\left(K_{0}(A)\right)$ onto $\tau_{B}\left(K_{0}(B)\right)$ can be extended to a group isomorphism $\varphi$ of $K_{0}(A)$ onto $K_{0}(B)$ in the way that

$$
\tau_{B} \circ \varphi=\tilde{\varphi} \circ \tau_{A} .
$$

This implies that $\varphi$ is an ordered isomorphism as well.

With a little more effort, we can use the same argument as above to prove the following result. Since we do not use it here, we state it without proof.

Proposition 7. If $A$ and $B$ are two non-rational $n$-tori and the rank of $\operatorname{ker}\left(\tau_{A}: K_{0}(A) \longmapsto \mathbf{R}\right)$ is not one, then

$$
\left(K_{0}(A), K_{0}^{+}(A),\left[1_{A}\right]\right) \cong\left(K_{0}(B), K_{0}^{+}(B),\left[1_{B}\right]\right)
$$

if and only if

$$
\tau_{A}\left(K_{0}(A)\right)=\tau_{B}\left(K_{0}(B)\right)
$$


Remark. It seems to be known that the range of the canonical trace on the $K_{0}$ group is not a complete invariant even for a simple non-commutative torus. This statement can be made more precise, not only by Proposition 6 but also by the following example. Let $q, p, p^{\prime}$ be positive integers such that

$$
(q, p)=\left(q, p^{\prime}\right)=1 \quad \text { and } \quad p \not \equiv \pm p^{\prime}(\bmod q) ;
$$

also let $\theta_{1}$ and $\theta_{2}$ be two real numbers such that $1, \theta_{1}, \theta_{2}$ are rationally independent. Let $\left\{U_{1}, U_{2}\right\}$ be a canonical unitary generator of $A_{p / q}$ and $\left\{\tilde{U}_{1}, \tilde{U}_{2}\right\}$ be a canonical unitary generator of $A_{p^{\prime} / q}$. Let $\alpha$ and $\tilde{\alpha}$ be automorphisms of $A_{p / q}$ and $A_{p^{\prime} / q}$ respectively given by

$$
\begin{aligned}
& \alpha\left(U_{1}\right)=\left(\exp 2 \pi i \theta_{1}\right) U_{1} \quad \text { and } \quad \alpha\left(U_{2}\right)=\left(\exp 2 \pi i \theta_{2}\right) U_{2} \\
& \tilde{\alpha}\left(\tilde{U}_{1}\right)=\left(\exp 2 \pi i \theta_{1}\right) \tilde{U}_{1} \text { and } \quad \tilde{\alpha}\left(\tilde{U}_{2}\right)=\left(\exp 2 \pi i \theta_{2}\right) \tilde{U}_{2} .
\end{aligned}
$$

Then $A=A_{p / q} \times{ }_{\alpha} \mathbf{Z}$ and $B=A_{p^{\prime} / q} \times_{\tilde{\alpha}} \mathbf{Z}$ are both simple and have the same range of the trace on the $K_{0}$-groups. But

$$
\left(K_{0}(A), K_{0}^{+}(A),\left[1_{A}\right]\right) \approx\left(K_{0}(B), K_{0}^{+}(B),\left[1_{B}\right]\right) .
$$

This last statement follows from the natural embedding of

$$
\left(K_{0}\left(A_{p / q}\right), K_{0}^{+}\left(A_{p / q}\right),\left[1_{A_{p / q}}\right]\right) \longmapsto\left(K_{0}(A), K_{0}^{+}(A),\left[1_{A}\right]\right),
$$

the strong Morita equivalence of $A_{p / q}$ with the commutative 2-torus (cf. Sect. 3 of [R2]) and the structure of all vector bundles over $\mathbf{T}^{2}$. The problem lies in the order unit.

Acknowledgements. This research was carried out at the Fields Institute for Research in Mathematical Sciences. I wish to acknowledge the support of G.A. Elliott and a helpful discussion with him. I also like to make a special acknowledgement to a helpful discussion with $\mathrm{H}$. $\mathrm{Su}$ and thank the referee for his helpful suggestions.

\section{References}

[B] Blackadar, B.: K-Theory for Operator Algebras. MSRI Pub. 5, New York: Springer, Berlin, Heidelberg, 1986

[Bo] Boca, F.: On the flip fixed point algebra in certain non-commutative tori. Preprint 1994

[Br] Bratteli, O.: Inductive limits of finite dimensional $\mathrm{C}^{*}$-algebras. Trans. Am. Math. Soc. 17, 195-234 (1972)

[BCEN] Brenken, B.A., Cuntz, J., Elliott, G.A., Nest, R.: On the classification of non-commutative tori, III. Operator Algebra and Mathematical Physics, Contemp. Math. 62, Providence, RI: Am. Math. Soc., 1987, pp. 503-526

[BGR] Brown, L., Green, P., Rieffel, M.A.: Stable isomorphism and strong Morita equivalence of $C^{*}$-algebras. Pacific J. Math. 71, 349-363 (1977)

[E] Elliott, G.A.: On the classification of $\mathrm{C}^{*}$-algebras of real rank zero. J. Reine Angew. Math. 443, 179-219 (1993)

[EE] Elliott, G.A., Evans, D.E.: The structure of the irrational rotation algebra. Ann. Math. 138, 477-501 (1993)

[EL1] Elliott, G.A., Lin, Q.: Cut-down method in the inductive limit decomposition of noncommutative tori. J. London Math. Soc., to appear 
[EL2] Elliott, G.A., Lin, Q.: Cut-down method in the inductive limit decomposition of noncommutative tori, II: The degenerate case, Operator Algebras and Their Applications, The Fields Institute Communications (1996)

[N] Newman, M.: Integral Matrices. New York: Academic Press, 1972

[P] Packer, J.A.: Transformation group $\mathrm{C}^{*}$-algebras: A selective survey, $\mathrm{C}^{*}$-algebras: 1943-1993 A Fifty Year Celebration, Contemp. Math. 157, Providence, RI: Amer. Math. Soc., 1994, pp. 183-217

[Pa] Park, C.: The bundle structure of non-commutative tori. Ph.D. dissertation (1995), University of Maryland

[Po] Poguntke, D.: The structure of twisted convolution $\mathrm{C}^{*}$-algebras on Abelian groups. Preprint 1994

[R1] Rieffel, M.A.: $C^{*}$-algebras associated with irrational rotations. Pacific J. Math. 93, 415-429 (1981)

[R2] Rieffel, M.A.: The cancellation theorem for projective modules over irrational rotation $\mathrm{C}^{*}$-algebras. Proc. London Math. Soc. 47, 285-302 (1983)

[R3] Rieffel, M.A.: Projective modules over higher dimensional non-commutative tori. Canad. J. Math. 40, 257-338 (1988)

[R4] Rieffel, M.A.: Non-commutative tori - A case study of non-commutative differentiable manifolds. Contemp. Math. 105, Providence, RI: Am. Math. Soc., 1990, pp. 191-211

[T] Takesaki, M.: Theory of Operator Algebras, I. New York: Springer, Berlin, Heidelberg, 1979.

Communicated by H. Araki 
